(C) 2020, The Authors. Published by Elsevier Inc. and Fass Inc. on behalf of the American Dairy Science Association ${ }^{\circledR}$. This is an open access article under the CC BY-NC-ND license (http://creativecommons.org/licenses/by-nc-nd/4.0/).

\title{
Randomized control trial assessing the efficacy of pain control strategies for caustic paste disbudding in dairy calves younger than 9 days of age
}

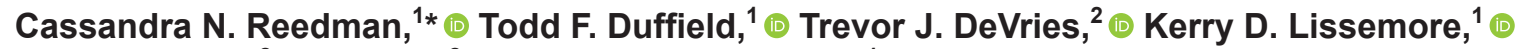 \\ Niel A. Karrow, ${ }^{2}{ }^{(}$Ziwei $^{2 i},{ }^{2}$ and Charlotte B. Winder ${ }^{1}\left({ }^{1}\right.$ \\ ${ }^{1}$ Department of Population Medicine, University of Guelph, Guelph, Ontario, N1G 2W1 Canada \\ ${ }^{2}$ Department of Animal Biosciences, University of Guelph, Guelph, Ontario, N1G 2W1 Canada
}

\begin{abstract}
Disbudding is a common procedure practiced in the dairy industry and is known to cause pain when performed without pain control. Dairy producers who disbud calves with caustic paste are less likely to provide pain control than those using cautery. Little research has been conducted on pain control for caustic paste disbudding and no studies have specifically examined calves under $9 \mathrm{~d}$ of age. The objective of this study was to evaluate the efficacy of local anesthesia and nonsteroidal anti-inflammatory drug analgesia on indicators of pain and inflammation in dairy calves disbudded using caustic paste. One hundred forty Holstein heifer calves 1 to $9 \mathrm{~d}$ of age were enrolled in 28 blocks and randomly allocated to 1 of 5 treatments: sham control (SH); positive control (POS); lidocaine cornual nerve block (LC); meloxicam (MEL); and lidocaine cornual nerve block plus meloxicam (LCM). We measured outcomes including serum cortisol and haptoglobin, pressure sensitivity, and lying behavior. Data were analyzed using mixed linear regression models with treatment as a fixed effect, baseline values as a covariate, and trial block as a random effect. Compared with the POS group, the LCM group had reduced serum cortisol at $15,30,45$, and 60 min post-disbudding; cortisol values were not different between $\mathrm{LC}, \mathrm{LCM}$, and $\mathrm{SH}$ calves at these time points. At 60, 90, 120, and 180 min postdisbudding, LCM calves had reduced cortisol compared with LC calves, whereas, values did not differ between LCM and SH calves at these time points. At 3 to 4 d post-disbudding, the LCM group tended to have reduced haptoglobin, but no differences were found between groups at $180 \mathrm{~min}$ and $7 \mathrm{~d}$ post-disbudding. At 60, 90, and 120 min post-disbudding, LC and LCM treated calves had decreased pressure sensitivity compared with other groups. No differences were seen in
\end{abstract}

Received December 23, 2019.

Accepted March 23, 2020.

*Corresponding author: creedman@uoguelph.ca pressure sensitivity between groups at $180 \mathrm{~min}, 3$ to 4 or $7 \mathrm{~d}$ post-disbudding. No differences in lying behavior were found between treatment groups on any of the $7 \mathrm{~d}$ following disbudding. These findings demonstrate that the combination of a local anesthetic with a nonsteroidal anti-inflammatory drug is beneficial for reducing indicators of pain and inflammation in young calves disbudded with caustic paste.

Key words: inflammation, analgesia, welfare, anesthesia

\section{INTRODUCTION}

Dehorning and disbudding are painful procedures commonly performed in the dairy industry (Stock et al., 2013). These procedures are performed for safety reasons because horned dairy cattle pose risk of injury to both people and other animals (Stock et al., 2013). Disbudding refers to the removal of the horn-forming tissue before approximately 2 mo of age and before attachment to the skull, while dehorning is the removal of the horn after this point (CVMA, 2016). Disbudding within the first month of life is recommended over dehorning because it is less invasive (CVMA, 2016; NFACC, 2009) and results in less pain and distress for the animal (Stafford and Mellor, 2005). Two main methods of disbudding are used: cautery and caustic paste. In Canada, $9 \%$ of producers report the use of caustic paste (Winder et al., 2018), while in the United States, $16 \%$ of producers report use of this method (NAHMS, 2014). In a study by Winder et al. (2018), it was also reported that of the producers using caustic paste as their primary method of disbudding, $73 \%$ disbudded their animals before they were 3 wk of age.

Caustic paste, or chemical disbudding, is the removal of the horn buds through a chemical burn typically caused by sodium and calcium hydroxide. Once the product is applied to the skin, it continues to burn the tissues until the paste has dried. Stilwell et al. (2009) demonstrated that the initial application of paste to the horn buds is not a painful process for the calf; however, 
indicators of pain were observed within the first $\mathrm{h}$ following paste application and for $3 \mathrm{~h}$ post-application. Therefore, the use of pain control is justified for the first $3 \mathrm{~h}$ post-disbudding.

Many researchers have demonstrated that when local anesthesia and a nonsteroidal anti-inflammatory drug (NSAID) are used together, they can decrease pain-related outcomes such as cortisol concentration, pressure sensitivity, and pain behaviors in response to disbudding (Faulkner and Weary, 2000; Duffield et al., 2010; Heinrich et al., 2010). Lying behaviors have also been reported to change in response to pain (Heinrich et al., 2010; Sutherland et al., 2018), and pain control has been reported to positively affect self-rewarding behaviors, such as play behaviors, following disbudding (Mintline et al., 2013). In a survey conducted among Ontario dairy producers, $62 \%$ of producers reported use of a local anesthetic and $24 \%$ reported use of a NSAID for disbudding procedures (Winder et al., 2016). It was also reported that compared with cautery, the use of caustic paste reduced the odds that the producer would provide pain control (Winder et al., 2016). One potential reason for this decreased use of pain control could be that an immediate avoidance reaction by calves is not observed during application of the paste (Stilwell et al., 2009), in contrast to application of a cautery iron. The provision of local anesthesia combined with a NSAID was demonstrated to be effective at reducing pain indicators in calves that were an average of $2 \mathrm{wk}$ (Winder et al., 2017) and 4 wk old (Stilwell et al., 2008) for caustic paste disbudding. Caustic paste is labeled for use on animals 2 mo of age and younger, including application instructions for animals under 1 wk of age (Dr Naylor Dehorning Paste, H. W. Naylor Co., Inc., Morris, NY).

The objective of this study was to evaluate the efficacy of $\mathrm{n}$ NSAID (meloxicam; MEL), local anesthetic (lidocaine; LC), and both medications given together $(\mathbf{L C M})$, as compared with no pain control (POS) and a sham control (SH), on outcomes associated with pain and inflammation (serum cortisol and haptoglobin concentrations, pressure sensitivity, and lying behavior) in dairy calves under $9 \mathrm{~d}$ of age. Cortisol concentrations were used to assess stress, haptoglobin concentrations to assess inflammation, algometry to measure the mechanical nociceptive threshold, or sensitivity, around the horn buds, and lying behaviors to examine potential behavioral differences between the treatment groups. We predicted that calves receiving LCM would have decreased cortisol concentrations, decreased haptoglobin concentrations, increased pressure sensitivity values, and fewer lying bouts following disbudding as compared with POS.

\section{MATERIALS AND METHODS}

This manuscript is reported according to guidelines for randomized control trials in livestock and food safety (REFLECT; O'Connor, 2010). An a priori trial protocol was published in the University of Guelph Institutional Repository on May 14, 2018, and is available at http://hdl.handle.net/10214/13043.

\section{Animal Use}

This trial was conducted between May and August of 2018 at a commercial dairy farm in southwestern Ontario. Use of animals and all methods for this study were approved by the University of Guelph Animal Care Committee (AUP\#4001) in compliance with animal use guidelines of the Canadian Council on Animal Care (1993).

\section{Housing and Management}

For the months of May, June, half of July, and half of August, calves were housed indoors in individual pens $(0.9 \times 2.1 \mathrm{~m})$ inside of a larger calf barn. Bedding consisted of wood shavings, which were replenished when calves moved to 2 calves per pen $(\sim 10 \mathrm{~d}$ old $)$ and replaced entirely between calves. These individual pens were closed off on 3 sides and had a metal gate at the front with 2 bucket attachments for water and starter feed. For half of the month of July and half of August, calves were housed in individual outdoor hutches (1.2 $\times 2.4 \mathrm{~m}$ ). Each outdoor hutch had a fence to create a small outdoor pen for each calf. Bedding inside the hutches consisted of wood shavings and bedding in the outdoor pens consisted of sand; these were also replaced between calves. Inside each hutch were 2 bucket holders for water and starter. Only heifer calves were used for the purposes of this trial. Calves were vaccinated with Once PMH (Merck, Kirkland, QC, Canada) at birth and $30 \mathrm{~d}$, and Bovi-Shield (Zoetis, Kirkland, QC, Canada) at $60 \mathrm{~d}$ of age. Navels were dipped with iodine solution at birth. Calves were moved from individual pens into group housing (maximum $\sim 15-20$ calves) at around 10 to $20 \mathrm{~d}$ of age, or when considered healthy enough.

\section{Nutrition}

At birth, calves were fed $4 \mathrm{~L}$ of colostrum split into two 2-L feedings within the first $6 \mathrm{~h}$ of life. Within $24 \mathrm{~h}$ of being born, calves were moved to individual pens where they were fed $3 \mathrm{~L}$ of milk twice daily until they reached approximately $20 \mathrm{~d}$ old; then they were 
increased to $4.5 \mathrm{~L}$ twice daily on mob feeders in groups of 10 calves. Calves were offered starter feed in a bucket from birth. Calves were weaned through a gradual weaning process beginning at 8 wk of age and were fully weaned from milk at 12 wk of age.

\section{Enrollment}

All heifer calves were health scored by researchers using the Calf Health Scorer (McGuirk, 2013) developed by the University of Wisconsin to determine their eligibility for the trial (McGuirk and Peek, 2014). Calves were eligible for trial if they were not appreciably polled by palpation of the horn buds and had health scores of $\leq 3$ for rectal temperature; $\leq 2$ for nasal discharge, ocular discharge, cough score, and fecal score; and $\leq 1$ for ear position, navel score, and joint score. Five calves were enrolled on each trial day, 1 for each treatment group. Only heifer calves $9 \mathrm{~d}$ of age or younger were considered for the trial.

One to 2 replicates were completed each week, depending on the number of healthy heifer calves born between trial days. Each replicate consisted of 5 calves randomly assigned to 1 of 5 treatments with each replicate containing all 5 treatments. Replicates were blocked by treatment group to account for any differences between treatment groups that could be explained by the day (e.g., weather, excessive noise).

\section{Treatment Groups}

The 5 treatment groups consisted of a sham group (SH), a paste group (positive control; POS), a paste + meloxicam group (MEL), a paste + lidocaine group (LC), and a paste + lidocaine and meloxicam group (LCM). Calves were restrained using a halter tied to the front of the pen for administering the cornual nerve blocks. The SH calves were given a saline cornual nerve block and noncaustic sham disbudding paste was applied (OmniVet Pharma Inc./Philco Animal Health Inc., Kitchener, ON, Canada); these calves were not disbudded during the study. The POS calves were given a saline cornual nerve block and disbudded with commercial caustic paste (calcium hydroxide $37.8 \%$, sodium hydroxide 24.9\%, Dr Naylor Dehorning Paste, H.W. Naylor Co., Inc.). The MEL calves were given a saline cornual nerve block and a subcutaneous injection of $0.5 \mathrm{mg} / \mathrm{kg}$ of meloxicam in the neck (Metacam 20 $\mathrm{mg} / \mathrm{mL}$ Solution for Injection, Boehringer Ingelheim, Burlington, ON, Canada). The LC calves were given a lidocaine cornual nerve block $(12 \mathrm{~mL}$ per calf, lidocaine hydrochloride injection $20 \mathrm{mg} / \mathrm{mL}$, Bimeda-MTC Animal Health Inc., Cambridge, ON, Canada). The LCM calves were given a lidocaine cornual nerve block and a subcutaneous injection of meloxicam. The MEL, LC, and LCM calves were all disbudded using commercial paste. Cornual nerve block technique was performed by insertion of an 18-gauge 1.5-inch needle caudal to the eye, injecting $6 \mathrm{~mL}$ per side fanned out in 3 directions as described in Winder et al. (2017). At -15 min relative to disbudding, depending on treatment, lidocaine or saline cornual nerve blocks and meloxicam were administered. Before any algometry measurements were taken, the hair around the horn buds of each calf was shaved using electric clippers. At the time of disbudding, the horn buds were scratched using steel wool to facilitate paste penetration and then disbudding was performed by creating a ring of petroleum jelly around each horn bud (to protect from spreading of the paste) and applying a thin layer of caustic paste. The same researcher for a given replicate used the fingertip of a gloved hand to apply the paste.

\section{Primary Outcome and Data Collection}

Experimental days occurred on Mondays and Fridays. Calves remained in their individual pens for the entirety of the experiment. Researchers arrived on farm around $0900 \mathrm{~h}$ to begin data collection, and paste application began at $1100 \mathrm{~h}$ each trial day.

Pressure sensitivity was measured via pressure force algometer (Force Ten FDX Compact Digital Force Gage, Wagner Instruments, Greenwich, CT) following the protocol described by Winder et al. (2017). Two trained researchers took these measurements with the same researcher collecting all measurements for each single trial day. The algometer was equipped with a rubber tip (1 cm in diameter). Measurements were taken at 4 different locations equidistant around each horn bud in the same order (Figure 1). With the calves restrained using a halter, the algometer was placed lightly on the site before force was fully applied until a withdrawal response from the calf. The sensitivity of the area was measured in kilograms-force (kgf) applied to each location and was referred to as the mechanical nociceptive threshold (MNT). Measurements for MNT were taken at $-25 \mathrm{~min}, 0,60 \mathrm{~min}, 90 \mathrm{~min}, 120$ min, 180 min, 3 or $4 \mathrm{~d}$, and $7 \mathrm{~d}$ relative to disbudding (Figure 2). The MNT measurements at the 4 locations around each horn bud were averaged to calculate 1 score for each calf at the different points in time. Minimum values were recorded at $0.5 \mathrm{kgf}$ and maximum values were recorded at $10 \mathrm{kgf}$. These responses included, but were not limited to, the calf jerking or shaking its head, pulling back sharply, jumping up or forward, and kicking. 


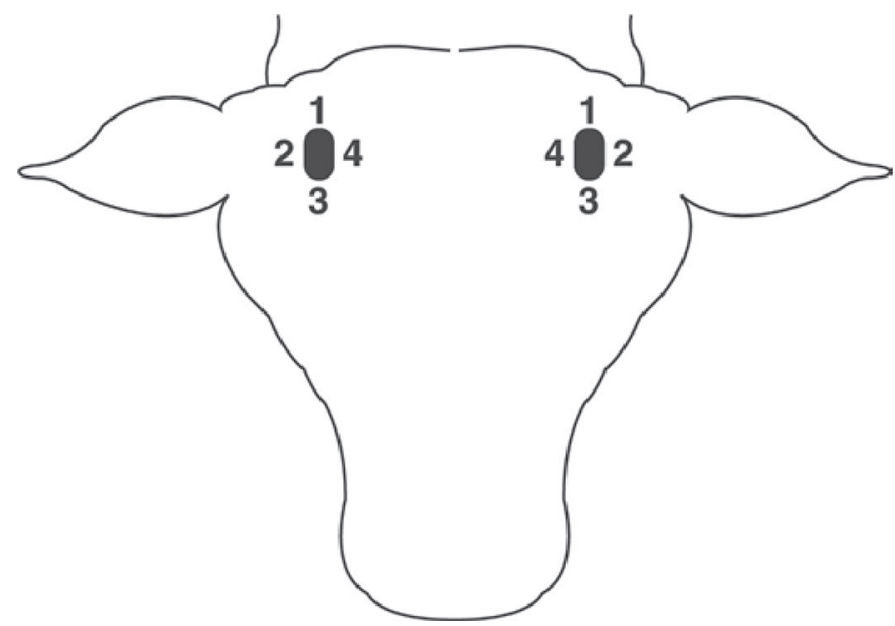

Figure 1. Locations around each horn bud where algometry was performed to measure pressure sensitivity. Sites are labeled with the order they were measured (Heinrich et al., 2010).

\section{Secondary Outcomes and Data Collection}

Serum Cortisol. Sixty minutes before disbudding occurred, a catheter was placed into the jugular vein on the left side of each calf with an extension set and held in place by sutures. Calves without a viable jugular vein on the left side had a catheter inserted into the vein on their right side. Following catheter insertion, each calf was allotted at least 30 min to rest and recover before more measures were taken. Samples for cortisol were obtained using red top vacutainer tubes via jugular catheter at $-25,0,15,30,45,60,90,120$, and $180 \mathrm{~min}$ relative to disbudding (Figure 2) and collected before other measures obtained at each sampling point. Before each vial of sample blood was taken, a discard tube was taken first to clear the catheter of saline-heparin solution. After blood samples were obtained, the catheter was flushed with the saline-heparin solution again. At $180 \mathrm{~min}$ relative to disbudding, catheters were removed from each calf and a bandage (composed of gauze and cohesive wrap) was applied to their neck. Calves were observed for 60 min following catheter removal to ensure proper healing, at which time the bandages were removed. At the end of each trial day, blood samples were centrifuged for $15 \mathrm{~min}$ at $1,500 \times g$. Samples for each time point were then separated into 4 microcentrifuge tubes and stored at $-80^{\circ} \mathrm{C}$ until time of testing. Cortisol samples were evaluated using a DetectX Cortisol Immunoassay kit (Arbor Assays, Ann Arbor, Michigan) to measure the amount of total cortisol present in the serum samples. A cortisol standard was provided to generate a standard curve for the assay to compare samples to. Cortisol samples were pipetted into a clear microtiter plate coated with an antibody to capture mouse antibodies; next, a cortisol-peroxidase conjugate was added and the binding reaction was initiated by the addition of a monoclonal antibody to cortisol. As the concentration of cortisol increases, the amount of cortisol-peroxidase conjugate bound decreases, causing a decrease in signal generated from the cortisol-peroxidase conjugate bound to the anti-cortisol antibody, which itself is bound to the goat anti-mouse IgG-coated plates. After $1 \mathrm{~h}$ of incubation, the plate was washed and the substrate was added. The substrate reacted with the bound cortisolperoxidase conjugate and the intensity of the generated color was detected in a microtiter plate reader (Cytation 5, BioTek, Winooski, VT) at $450 \mathrm{~nm}$ wavelength. The lowest detectable concentration was $45.4 \mathrm{pg} / \mathrm{mL}$, and the intra- and inter-assay coefficients of variation were $7.7 \%$ and $3.4 \%$, respectively.

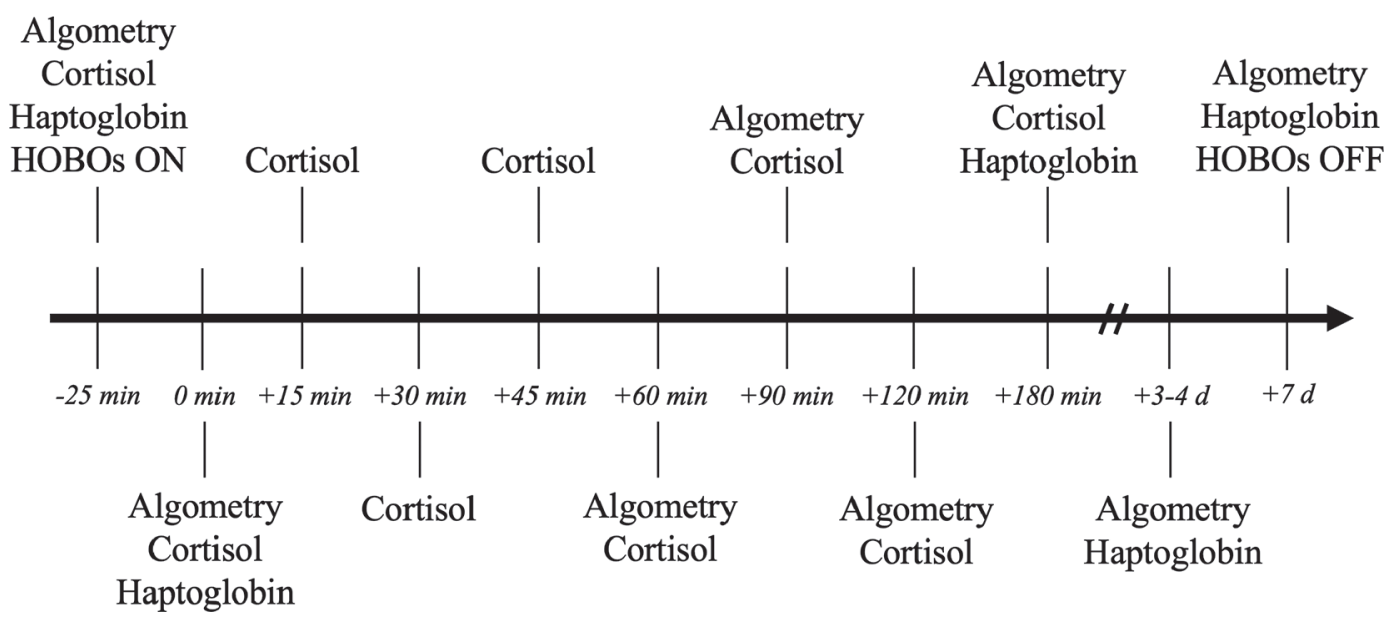

Figure 2. Timeline displaying data collection for all outcomes relative to disbudding. HOBOs ON/OFF refers to start and end of data collection using HOBO data loggers (Onset Computer Corp., Bourne, MA). 
Standing and Lying Bouts. For measurements of standing and lying bouts, we used HOBO Pendant G Data Loggers (Onset Computer Corp., Bourne, MA). Loggers were set to record every $60 \mathrm{~s}$ and were attached horizontally to the rear right leg of each calf, as described and validated by Bonk et al. (2013), using a cohesive bandage on the day of experimental treatment 90 min before disbudding. Loggers remained attached to each calf for the duration of the trial and for an additional $6 \mathrm{~d}$ (Figure 2). No baseline values were collected for this outcome as the data loggers were attached following enrollment, but before treatment on the day of disbudding. Data were downloaded using HOBOware Pro Software (Onset Computer Corp.), imported into Microsoft Excel (Microsoft Corp., Redmond, WA), and categorized into 3 outcomes: average time spent lying in a day, average lying bout length, and number of lying bouts in a day.

Serum Haptoglobin. Blood samples for haptoglobin were taken using red top vacutainer tubes via jugular catheter at $-25 \mathrm{~min}, 0$, and $180 \mathrm{~min}, 3$ to $4 \mathrm{~d}$, and 7 $\mathrm{d}$ relative to disbudding (Figure 2). Samples taken the day of disbudding were obtained as described above, and additional samples collected at d 3 to 4 and d 7 were obtained by jugular venipuncture. Blood samples were centrifuged for $15 \mathrm{~min}$ at $1,500 \times \mathrm{g}$. Serum from each sampling time was separated, harvested, and stored at $-20^{\circ} \mathrm{C}$ until time of testing. Haptoglobin samples were run as a single batch on a Roche Cobas 6000 c501 biochemistry analyzer (Roche Diagnostics, Laval, QC, Canada) using a methemoglobin stock reagent with formulas and operating conditions developed by the J. G. Skinner laboratory, Veterinary Investigation Centre (Aberdeen, UK; Makimura and Suzuki, 1982; Skinner et al., 1991). Methemoglobin combines with haptoglobin to form a stable methemoglobin-haptoglobin complex; measurement of haptoglobin is based on the peroxidase activity of this complex in acidic conditions. A hydrogen peroxide and guaiacol solution served as the substrate for the reaction, and color development was read at $480 \mathrm{~nm}$ wavelength. The inter-assay coefficient of variation was $4.8 \%$.

\section{Sample Size}

The sample size was calculated a priori, considering algometry as a primary outcome based on an expected difference of $0.5 \mathrm{kgf}$ between treatment groups (1.0 vs. $1.5 \mathrm{kgf}$ ) with a standard deviation of $0.5 \mathrm{kgf}, 95 \%$ confidence, and $80 \%$ power, based on findings from Heinrich et al. (2010) and Winder et al. (2017). The calculated sample size per treatment group after adjusting for clustering was 24 calves, which we rounded up by 4 per group to account for possible post hoc exclusions.

\section{Treatment Allocation and Blinding}

Calves in this study were blocked by treatment; each trial day included all 5 treatments given to 5 heifer calves. If more than 5 heifer calves were born between visits, the first 5 that passed the screening protocol were used, with a preference for a calf from each potential birth date fitting the criteria (i.e. $1 \mathrm{~d}$ old, $2 \mathrm{~d}$ old, 3 $\mathrm{d}$ old, $4 \mathrm{~d}$ old, and $5 \mathrm{~d}$ old). Treatments were assigned randomly to calves by blindly shuffling 5 cards containing a letter for each treatment (A, B, C, D and E). Treatments were assigned after all calves were health scored, had met trial criteria, and were enrolled in the trial. A member of the research team who was blind to all treatments drew the treatment cards. The research team, including the farm staff and those involved in treatment administration, data collection, and evaluation and analysis, were blind to the LC treatment through the use of lettered, identical containers holding either lidocaine or saline, and caustic or sham paste. The MEL treatments were blinded to all individuals involved, with the exception of 1 research student who knew which 2 letters indicated meloxicam to administer the product; this student was not involved in further treatment administration, evaluation of outcomes, or analysis.

\section{Statistical Analysis}

All recorded data were entered into Microsoft Excel and imported into STATA15 (Stata/IC Version 15.1 for Mac, StataCorp, College Station, TX). Descriptive statistics were reviewed for all variables. All variables were also assessed for normality, linearity, and variation through assessment of outliers and residuals.

For outcomes with repeated measures (algometry, serum cortisol, and serum haptoglobin), a mixed model was built for each outcome with baseline measures as a covariate, and calf nested within day as a random effect. For continuous, normally distributed data (MNT, serum cortisol, and serum haptoglobin), linear models were used. The effect of treatment on outcome was tested, as well as first-order interaction terms for treatment by time, and calf age were offered to each model. If a significant $(P<0.05)$ treatment by time interaction was detected (MNT, serum cortisol, and serum haptoglobin), the effect of treatment on outcome was stratified by time, with single-level models built for each time point.

For standing and lying data, daily average time spent lying, average lying bout length, and number of lying bouts per day were assessed as separate outcomes using mixed models with calf nested within day as a random effect. For continuous, normally distributed data (daily 
Table 1. Number of samples included in analysis and missing data by outcome for each time point collected

\begin{tabular}{|c|c|c|c|}
\hline Outcome & $\begin{array}{l}\text { Time relative } \\
\text { to disbudding }\end{array}$ & $\begin{array}{l}\text { Number } \\
\text { included }\end{array}$ & $\begin{array}{c}\text { Number } \\
\text { missing }\end{array}$ \\
\hline \multirow[t]{8}{*}{ Algometry } & $-25 \min$ & 140 & 0 \\
\hline & $0 \mathrm{~min}$ & 140 & 0 \\
\hline & $60 \min$ & 139 & 1 \\
\hline & $90 \mathrm{~min}$ & 140 & 0 \\
\hline & $120 \min$ & 140 & 0 \\
\hline & $180 \mathrm{~min}$ & 140 & 0 \\
\hline & $3-4 \mathrm{~d}$ & 134 & 6 \\
\hline & $7 \mathrm{~d}$ & 130 & 10 \\
\hline \multirow[t]{9}{*}{ Cortisol } & $-25 \min$ & 140 & 0 \\
\hline & $0 \min$ & 140 & 0 \\
\hline & $15 \mathrm{~min}$ & 138 & 2 \\
\hline & $30 \mathrm{~min}$ & 138 & 2 \\
\hline & $45 \min$ & 139 & 1 \\
\hline & $60 \mathrm{~min}$ & 140 & 0 \\
\hline & $90 \min$ & 140 & 0 \\
\hline & $120 \mathrm{~min}$ & 140 & 0 \\
\hline & $180 \mathrm{~min}$ & 140 & 0 \\
\hline \multirow[t]{5}{*}{ Haptoglobin } & $-25 \min$ & 140 & 0 \\
\hline & 0 min & 140 & 0 \\
\hline & $180 \min$ & 140 & 0 \\
\hline & $3-4 \mathrm{~d}$ & 140 & 0 \\
\hline & $7 \mathrm{~d}$ & 140 & 0 \\
\hline Standing or lying & All data from $7 \mathrm{~d}$ & 127 & 13 \\
\hline
\end{tabular}

average time spent lying, average lying bout length, and number of lying bouts per day), linear models were used. Effect of treatment, calf age, and day on outcome were tested along with first-order interaction terms between these variables. No significant treatment by time interactions were found.

\section{RESULTS}

In total, 140 calves were enrolled in this study with 28 calves randomly assigned to each of the 5 treatment groups. All enrolled calves received the intended treatment depending on their group and were followed for $7 \mathrm{~d}$. All samples and measurements collected were used for analysis. Missing data are described in Table 1. No deviations from the trial protocol occurred for treatment allocation, randomization, data collection, or data analysis.
Calf age was found to be significant for models assessing cortisol and haptoglobin, although after further investigation was not included in the final models because baseline values were included as a fixed effect. Age was found to be significant in the models assessing baseline value as well as the other time points following disbudding, but was no longer significant in the models once baseline values were controlled for. Therefore, we included baseline values as a covariate to control for differences between groups and did not need to control for age.

\section{Baseline Characteristics}

Calf age ranged from 1 to $9 \mathrm{~d}$ on the day of disbudding. The mean age did not differ between the treatment groups $(3.7 \pm 0.16 \mathrm{~d} ; P>0.2)$. Baseline values for each outcome are described in Table 2 stratified by treatment group. Values for MNT, cortisol, and haptoglobin were not statistically different between treatment groups $(P>0.05)$.

\section{Primary Outcomes}

The MNT scores over time by treatment group are described in Figure 3. At 0 min, immediately before caustic or sham paste was applied, the LC and LCM groups had greater MNT values than the other 3 treatment groups $(P<0.001)$, meaning they were less sensitive and able to withstand more force applied to the areas around their horn buds (Figure 1). At 60 and 90 min following paste application, the LC and LCM groups had greater MNT values than the other 3 treatment groups $(P<0.01)$. At 120 min following paste application, the LCM group had greater MNT values than the POS, MEL, and SH groups $(P<0.05)$, and the LC group had greater MNT scores than the MEL group (0.90 kgf, $95 \%$ CI 0.10 to $1.69, P=0.03)$. At 0,90 , and 120 min there were no detected differences between the POS, MEL, and SH treatment groups. At $180 \mathrm{~min}, 3$ to $4 \mathrm{~d}$, and $7 \mathrm{~d}$ following paste application there were no detected differences between any of the 5 treatment groups.

Table 2. Baseline characteristics for age, algometry, cortisol, and haptoglobin stratified by treatment group (mean \pm SD)

\begin{tabular}{|c|c|c|c|c|c|}
\hline Item & $\begin{array}{c}\text { Sham } \\
(\mathrm{n}=28)\end{array}$ & $\begin{array}{l}\text { No pain control } \\
\quad(\mathrm{n}=28)\end{array}$ & $\begin{array}{l}\text { Meloxicam } \\
\quad(\mathrm{n}=28)\end{array}$ & $\begin{array}{l}\text { Lidocaine } \\
(\mathrm{n}=28)\end{array}$ & $\begin{array}{c}\text { Meloxicam and } \\
\text { lidocaine }(\mathrm{n}=28)\end{array}$ \\
\hline Age $(d)$ & $3.3( \pm 1.8)$ & $4.0( \pm 1.7)$ & $4.1( \pm 1.9)$ & $3.3( \pm 2.0)$ & $4.0( \pm 1.9)$ \\
\hline Algometry $\left(\mathrm{kgf}^{1}\right)$ & $5.2( \pm 1.9)$ & $5.2( \pm 1.7)$ & $4.8( \pm 1.8)$ & $5.2( \pm 1.9)$ & $5.3( \pm 2.1)$ \\
\hline Haptoglobin $(\mathrm{mg} / \mathrm{mL})$ & $0.14( \pm 0.03)$ & $0.22( \pm 0.30)$ & $0.19( \pm 0.15)$ & $0.23( \pm 0.22)$ & $0.26( \pm 0.32)$ \\
\hline
\end{tabular}

${ }^{1}$ Kilograms-force. 


\section{Secondary Outcomes}

Serum Cortisol. Cortisol results by treatment group are described in Figure 4. At 0 min, directly before the caustic or sham paste was applied, there were no significant differences between any of the treatment groups $(P>0.05)$. At $15 \mathrm{~min}$, the POS group had greater cortisol concentrations than the SH group (95\% CI 19.1 to $136.0, P<0.01)$. At 30,45 , and $60 \mathrm{~min}$ post-disbudding, the POS group had greater cortisol concentrations than the SH group $(P<0.01)$. At 120 min, the POS group was not different than the $\mathrm{SH}$ group (95\% CI -43.9 to $46.9, P=0.95)$, and at 180 min the POS group had lesser cortisol concentrations than the SH group (95\% CI -106.9 to $-4.5, P=0.03)$.

Efficacy of Meloxicam Alone. At 15 and 30 min, the MEL group had greater cortisol concentrations compared with the SH group $(P<0.05$; Figure $4)$ and was not different than the POS group $(P>$ 0.05). At 45 min the MEL group had greater cortisol concentrations than the SH group $(79.0 \mathrm{pg} / \mathrm{mL}, 95 \%$ CI 25.3 to $132.7 ; P<0.01$; Figure 4$)$ and had lesser cortisol concentrations than the POS group $(-57.2 \mathrm{pg} /$ $\mathrm{mL}, 95 \% \mathrm{CI}-111.4$ to $-2.9 ; P=0.04 ;$ Figure 4$)$. At 60 min following disbudding, the MEL group had greater cortisol concentrations than the $\mathrm{SH}$ group $(64.5 \mathrm{pg} /$
$\mathrm{mL}, 95 \%$ CI 3.8 to $125.2 ; P=0.04$; Figure 4 ) and were not different from the POS group. At $90 \mathrm{~min}$, the MEL group had lesser cortisol concentrations than the POS group $(-67.8 \mathrm{pg} / \mathrm{mL}, 95 \%$ CI -118.3 to $-17.3 ; P<$ 0.01; Figure 4); concentrations did not differ between the MEL and SH groups at this time.

Efficacy of Lidocaine Alone. At 15, 30, and 45 min, the LC group had lesser cortisol values than the MEL and POS groups $(P<0.05$; Figure 4$)$. Additionally, concentrations were not detected different between the LC and $\mathrm{SH}$ groups at this time $(P>0.05)$. At 60 min, the LC group had lesser cortisol concentrations than the POS group $(-76.2 \mathrm{pg} / \mathrm{mL}, 95 \% \mathrm{CI}-137.5$ to $-15.0 ; P=0.02$; Figure 4). At 90 and $120 \mathrm{~min}$, the LC group had greater cortisol concentrations than the $\mathrm{SH}$ and MEL groups $(P<0.05$; Figure 4$)$. At $180 \mathrm{~min}$, the LC group had greater cortisol concentrations than the POS and MEL groups $(P<0.01$; Figure 4$)$.

Efficacy of Lidocaine and Meloxicam. At 15 and $30 \mathrm{~min}$, the LCM group had lesser cortisol concentrations than the POS, MEL, and SH groups $(P<$ 0.05 ; Figure 4$)$, and concentrations were not different between the LCM group and LC groups $(P>0.05)$. At $45 \mathrm{~min}$, the LCM group had lesser cortisol concentrations than the POS and MEL groups $(P<0.001$; Figure 4), and concentrations were not different between

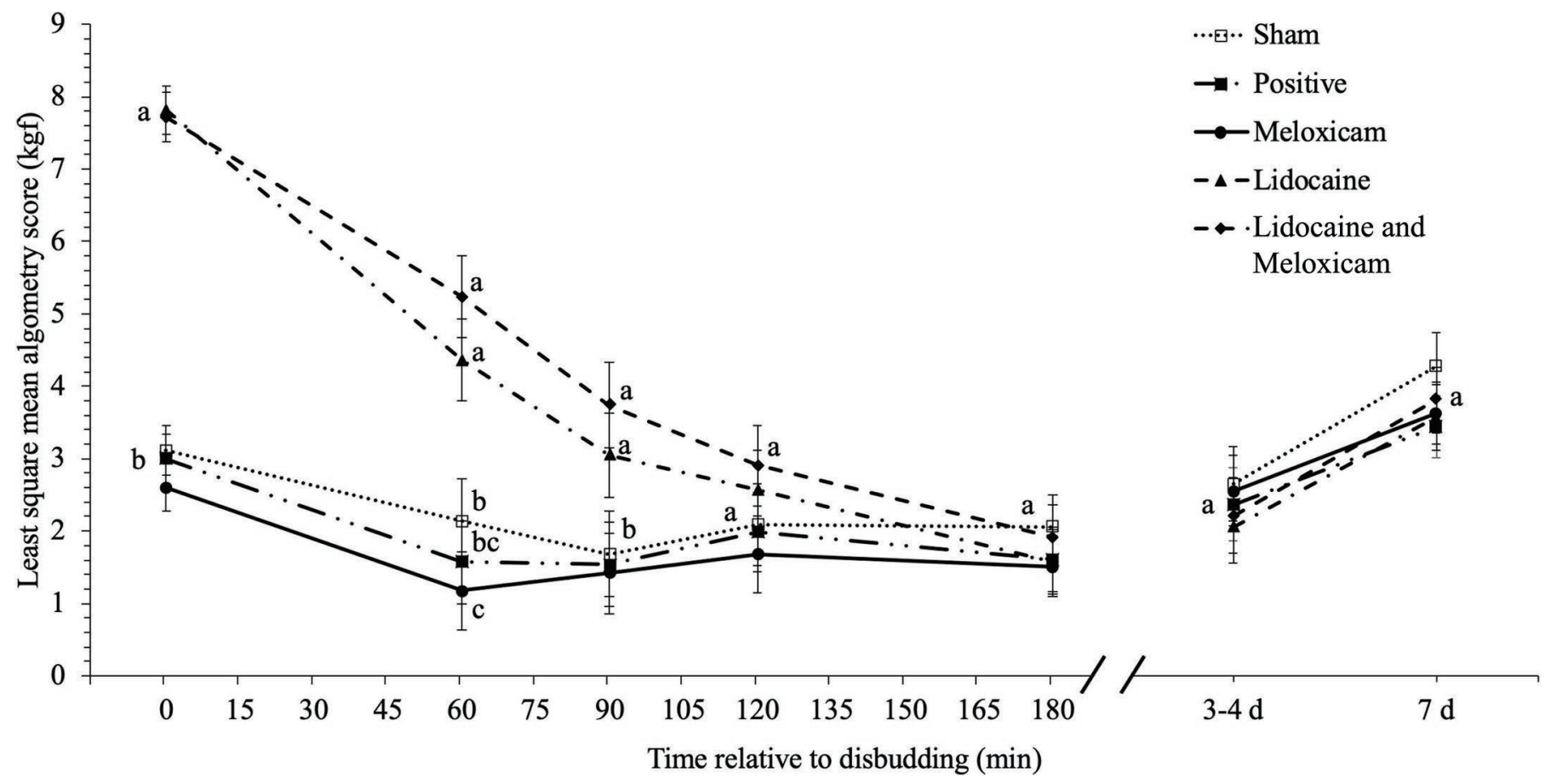

Figure 3. Least squares means $( \pm \mathrm{SE})$ algometry scores of 5 treatment groups over time relative to disbudding with caustic or sham paste. All groups received the saline or lidocaine cornual nerve block and subcutaneous injection of meloxicam (if applicable) 15 min before caustic paste was applied. Different letters $(\mathrm{a}-\mathrm{c})$ represent significant differences $(P<0.05)$ between treatment groups for a single time point. kgf $=$ kilograms-force. 
the LCM group and the LC group (95\% CI -10.7 to 95.8, $P=0.12$ ). At 45, 60, 90, 120, and $180 \mathrm{~min}$, there were no detected differences in cortisol concentrations between the LCM and $\mathrm{SH}$ groups $(P>0.05)$. At 60 min, the LCM group had lesser cortisol concentrations than the POS, MEL, and LC groups $(P<0.05$; Figure 4). At 60, 90, 120, and $180 \mathrm{~min}$, the LCM group had lesser cortisol concentrations than the LC group $(P<$ 0.05; Figure 4). At $90 \mathrm{~min}$, the LCM group had lesser cortisol concentrations than the POS and LC groups $(P<0.05 ;$ Figure 4$)$, and at 120 and $180 \mathrm{~min}$, cortisol concentrations did not differ between the LCM group and the POS and MEL groups $(P>0.05)$.

Serum Haptoglobin. No significant differences were found between any of the treatment groups at 0 min, 180 min, or 6 to $7 \mathrm{~d}$. At 3 or $4 \mathrm{~d}$, the LCM group tended to have lesser haptoglobin concentrations than the POS group $(-0.16 \mathrm{mg} / \mathrm{mL}, 95 \%$ CI -0.32 to 0.00 ; $P=0.05)$. We detected no other differences between other treatment groups at this time point.

Standing and Lying Bouts. No treatment by time interactions were detected for any of the outcomes assessed. On d 1 (day of disbudding), regardless of treatment group, calves had a decreased average lying bout length $(P<0.05)$ and an increased number of lying bouts compared with the other $6 \mathrm{~d}$ following disbudding $(P<0.05)$. For each outcome, we found no discernable pattern or differences between treatment groups on the day of disbudding or any of the 6-d following disbudding.

\section{DISCUSSION}

In this study, we demonstrated that without the use of either local anesthesia, a NSAID, or the combination of both, chemical disbudding with caustic paste is acutely painful for young calves for at least $3 \mathrm{~h}$ following disbudding, similar to patterns that have been reported in other work (Morisse et al., 1995; Stilwell et al., 2008; Winder et al., 2017). In contrast to other studies examining older calves (2 wk old in Winder et al., 2017; 9 wk old in Heinrich et al., 2010), our MNT results did not show the same differences between treatment groups as our cortisol results, indicating that this behavioral test may be less reliable for younger calves.

\section{Physiological Response}

Based on our results, cortisol concentrations showed a similar pattern to other work assessing pain post-

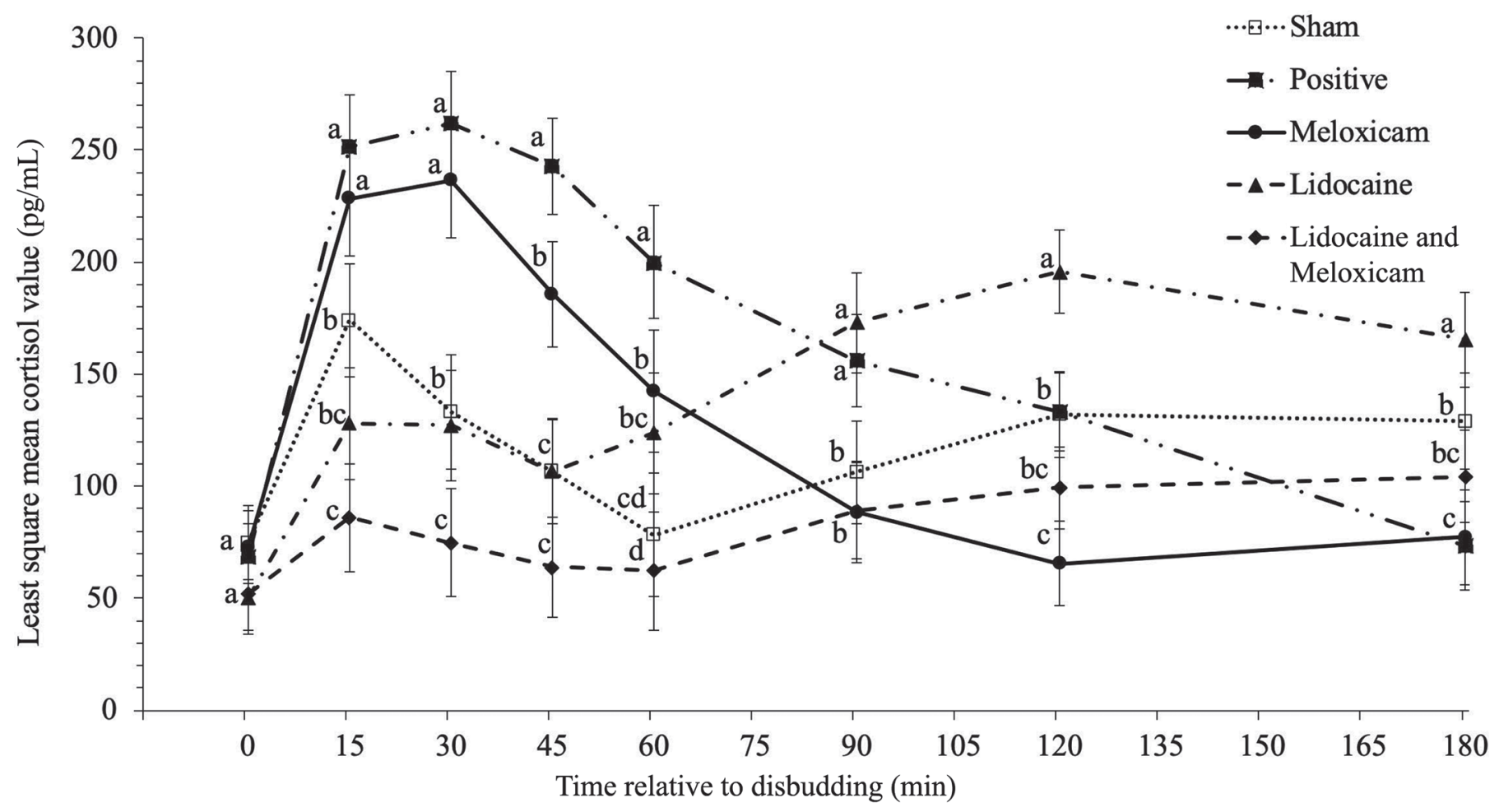

Figure 4. Least squares means $( \pm \mathrm{SE})$ serum cortisol value of 5 treatment groups over time relative to disbudding with caustic or sham paste. All groups received the saline or lidocaine cornual nerve block and subcutaneous injection of meloxicam (if applicable) 15 min before caustic paste was applied. Different letters $(\mathrm{a}-\mathrm{d})$ represent significant differences $(P<0.05)$ between treatment groups for a single time point. 
disbudding (Petrie et al., 1996; Mellor et al., 2002; Stilwell et al., 2009). Our results demonstrated that a local anesthetic (lidocaine) was successful at mitigating acute pain from the procedure, whereas a longer term NSAID (meloxicam) was beneficial at reducing inflammatory pain. The MEL group showed a peak in cortisol concentration in the first 30 min following disbudding (Figure 4), which is consistent with results reported by McMeekan et al. (1998) assessing the use of ketoprofen alone for scoop dehorning calves. The meloxicam may mitigate some of the inflammatory pain seen at 90 min post-disbudding, as cortisol concentration for the MEL group were lower than that of the POS group at several time points following paste application. Cortisol is known to be a powerful anti-inflammatory hormone (Hannibal and Bishop, 2014); cortisol for the MEL and POS groups began decreasing to basal concentrations between 90 and $120 \mathrm{~min}$, indicating that the initial spike in cortisol suppressed the inflammation observed later on. Numerous other studies have shown the return of cortisol to basal concentrations following a spike shortly after disbudding (Morisse et al., 1995; Sutherland et al., 2002; Stilwell et al., 2008).

The steady increase of cortisol concentrations that we observed at 60 to 90 min following the provision of a local anesthetic alone has been reported in research assessing scoop dehorning (Petrie et al., 1996; McMeekan et al., 1998), as well as caustic paste disbudding, at 2 to $4 \mathrm{~h}$ following the procedure (Stilwell et al., 2009), and cautery disbudding at 3 to $6 \mathrm{~h}$ post-disbudding (Stilwell et al., 2012). In our study, the LC group displayed a protracted rise in cortisol concentrations at 90 min post-disbudding once the lidocaine had worn off, while the LCM group did not show this same increase. Previous work assessing the cortisol response reported similar results; calves provided with both a local anesthetic and analgesic did not display an initial or delayed cortisol concentration increase when compared with a local anesthetic alone in both scoop dehorning (Sutherland et al., 2002) and cautery disbudding (Milligan et al., 2004; Stilwell et al., 2012). Similar to cautery disbudding, these results highlight the importance of providing both local anesthesia and analgesia to mitigate the acute and inflammatory pain responses associated with caustic paste disbudding in very young calves. Few studies have reported differences in pain between cautery and caustic paste disbudding (Morisse et al., 1995; Vickers et al., 2005), but no studies have examined differences between these methods providing both a local anesthetic and analgesic as the primary objective.

Haptoglobin is an acute phase protein and a biomarker of inflammation in cattle (Makimura and Su- zuki, 1982). Haptoglobin sampling for this study was limited to the days that researchers were on farm, and therefore could only be collected on the day of disbudding, 3 or $4 \mathrm{~d}$ later, and $7 \mathrm{~d}$ later. In a study by Ballou et al. (2013), the researchers reported lower haptoglobin concentrations in calves that were scoop dehorned with an analgesic compared with no analgesic $1 \mathrm{~d}$ post-disbudding, but observed no differences at $3 \mathrm{~d}$ post-disbudding. In contrast, Hempstead et al. (2018) reported no difference in haptoglobin concentrations at $1 \mathrm{~d}$ post-disbudding compared with baseline values in goat kids that were disbudded with caustic paste. In our study, we were unable to obtain samples for haptoglobin at $1 \mathrm{~d}$ post-disbudding, but at 3 or $4 \mathrm{~d}$ postdisbudding, calves in the LCM group tended to have lower haptoglobin levels compared with the POS group. Due to our limitations in sampling, we were unable to detect any differences between treatment groups that may have been present on other days post-disbudding. The results on 3 or $4 \mathrm{~d}$ post-disbudding suggest that treatment with both a local anesthetic and analgesic could aid in reducing inflammation in young calves disbudded with caustic paste. Further research assessing daily haptoglobin concentrations in the context of disbudding research would be beneficial in understanding the role of haptoglobin and inflammation following these procedures.

\section{Mechanical Nociceptive Threshold}

Based on our results, it appears that calves that received lidocaine (with or without meloxicam) were desensitized for the first $1.5 \mathrm{~h}$ following disbudding, consistent with other studies that assessed pressure sensitivity following disbudding procedures (Heinrich et al., 2010; Espinoza et al., 2013; Winder et al., 2017).

Mechanical nociceptive threshold is commonly used to assess pain sensitivity in disbudding research, especially in studies involving cautery disbudding (Stock et al., 2015; Heinrich et al., 2010; Stock et al., 2016). Less research has been conducted surrounding disbudding via caustic paste, although in a study by Winder et al. (2017), it was reported that calves treated with lidocaine had higher MNT scores when compared with other treatment groups. In contrast to what we detected, Winder et al. (2017) reported that the positive control group (compared with the sham control group), had much lower MNT values after 30 min following disbudding. We did not detect any differences between the SH group and the POS and MEL groups for any point in time following disbudding. These results could indicate that these calves did not respond to the test because they were not in pain, although our cortisol 
results indicate otherwise. Our results are also different than previous work assessing MNT in both cautery (Stock et al., 2016; Adcock and Tucker, 2018; Stock et al., 2015) and caustic paste disbudding (Winder et al., 2017) in older calves (2-8 wk). Our methodology for assessing MNT was very similar to that used by Winder et al. (2017; 2-wk-old calves) and Heinrich et al. (2010; 9 -wk-old calves), but differences between our results and results reported from Adcock and Tucker (2018; 7-wk-old calves) could potentially be due to a difference in methodology and restraint method employed for MNT measurements. Although similar to what we detected, in a study assessing pain following caustic paste disbudding in 3-d-old calves, Karlen et al. (2019) reported that they did not detect differences in mean MNT values between calves that were not provided with any pain mitigation and sham disbudded calves from 12 to $300 \mathrm{~h}$ following paste application. From accumulated results, it appears that younger calves (1-9 d old) might not react to the MNT test in the same way that older calves (2-9 wk) do, making it difficult to use this test as a measure of pressure sensitivity in younger calves. The lack of withdrawal response from the young calves disbudded with no pain control in this study, as well as in the study by Karlen et al. (2019), could indicate that calves of this age have not developed this response yet, suggesting behavioral differences between calves less than $9 \mathrm{~d}$ of age and 2- to 9-wk-old calves. However, little research has examined behavioral differences between young and old calves. Young calves (in their first week of life) display increased hiding behavior compared with older calves (Langbein and Raasch, 2000). Calves spend more time hiding when out at pasture at $1 \mathrm{~d}$ old, with time spent hiding decreasing from 1 to $5 \mathrm{~d}$ of age (Langbein and Raasch, 2000). Further research assessing the potential differences in the behavior of young calves compared with older calves could help in understanding why the algometry test provided different results than the cortisol responses in these calves. Additionally, further work assessing MNT in young calves could help explain why these results differ compared with work in older calves.

\section{Standing and Lying Behavior}

Standing and lying behaviors were assessed as number of lying bouts per day, average lying bout length, and average daily lying time. There were no detected differences between treatment groups for any of these outcomes. In the work by Sutherland et al. (2018), it was reported that following cautery disbudding, sham control calves, calves treated with a local anesthetic alone, and calves treated with a local anesthetic and a NSAID spent more time lying in the first hour post- disbudding than compared with calves disbudded with no pain control and a NSAID alone, but no differences were observed between groups at 2 or $24 \mathrm{~h}$ post-disbudding. Our results demonstrated that all 5 treatment groups had shorter average lying bouts and average daily lying time on d 1 (the day disbudding occurred), but this is most likely a result of researcher presence in the barn and calve movement associated with collecting algometry measurements and blood samples. A potential reason for the lack of differences found in this study could be due to the different housing conditions throughout the summer, with calves housed outdoors being provided with more space than the calves housed indoors. Further research assessing lying behavior postdisbudding without collecting other measures would be beneficial in understanding the role of pain control methods on these behaviors.

Other studies have used established pain behaviors such as ear flicking, head shaking, and scratching or rubbing as behavioral indicators of pain following both cautery (Heinrich et al., 2010) and caustic paste disbudding (Stilwell et al., 2009). These behaviors have been reported to be present immediately following caustic paste disbudding in 3 to 4 wk old calves disbudded with no pain mitigation (Stilwell et al., 2009). We were limited in this study by the number of researchers available to collect data. This was a labor-intensive study, and we were therefore unable to collect data on pain behaviors following the procedure. Work assessing these behaviors in young calves $(<1$ wk of age) following caustic paste disbudding would be beneficial in understanding the effect of treatment on these behaviors.

\section{Age and Baseline Values}

This study did not have the statistical power to examine the effects of calf age on the assessed outcomes. Older calves and younger calves in this study had different physiological baselines for cortisol and haptoglobin concentrations and could respond to MNT tests differently as well. Age was identified as a significant variable in the models built assessing algometry, cortisol, and haptoglobin at the time points following disbudding. However, it was also found to be significant when assessing the baseline values for all of these outcomes. Therefore, including baseline values as a covariate for the models assessing these outcomes, we can control for any differences that may be attributable to age. Further research assessing the effect of age, by enrolling a higher number of older calves to compare with the young calves, may help in understanding differences that could be attributed to age or MNT differences between older and younger calves. 


\section{CONCLUSIONS}

The results of this study indicate that the combination of a local anesthetic and a NSAID is beneficial at reducing indicators of pain and inflammation in very young dairy calves disbudded with caustic paste. Discrepancies between cortisol and algometry results might indicate that this MNT test may be less reliable for younger calves disbudded with caustic paste than it is for older calves. We recommend providing both a local anesthetic and a NSAID when performing caustic paste disbudding on dairy calves $9 \mathrm{~d}$ of age and younger.

\section{ACKNOWLEDGMENTS}

The authors thank the dairy farm, all of the farm staff, and research students for their contributions to this work. This project was funded by the Ontario Ministry of Agriculture, Food, and Rural Affairs-University of Guelph Ontario Agri-Food Innovation Alliance Research Program (Guelph, ON, Canada), BoehringerIngelheim (Burlington, ON, Canada), Dairy Farmers of Ontario (Mississauga, ON, Canada), and Saputo Dairy Care Program (Guelph, ON, Canada). The authors have not stated any conflicts of interest.

\section{REFERENCES}

Adcock, S. J. J., and C. B. Tucker. 2018. The effect of disbudding age on healing and pain sensitivity in dairy calves. J. Dairy Sci. 101:10361-10373. https://doi.org/10.3168/jds.2018-14987.

Ballou, M. A., M. A. Sutherland, T. A. Brooks, L. E. Hulbert, B. L. Davis, and C. J. Cobb. 2013. Administration of anesthetic and analgesic prevent the suppression of many leukocyte responses following surgical castration and physical dehorning. Vet. Immunol. Immunopathol. 151:285-293. https://doi.org/10.1016/j.vetimm .2012.11.018.

Bonk, S., O. Burfeind, V. Suthar, and H. Heuwieser. 2013. Technical note: Evaluation of data loggers for measuring lying behaviour in dairy calves. J. Dairy Sci. 96:3265-3271. https://doi.org/10.3168/ jds.2012-6003.

Canadian Council on Animal Care. 1993. Guide to the Care and Use of Experimental Animals. Vol. 1, 2nd ed. Accessed December 11th, 2019. https://www.ccac.ca/Documents/Standards/Guidelines/ Experimental_Animals_Vol1.pdf

Canadian Veterinary Medical Association. 2016. Disbudding and dehorning of cattle - Position statement. Accessed October 1st, 2019. https://www.canadianveterinarians.net/documents/disbudding -and-dehorning-of-cattle

Duffield, T. F., A. Heinrich, S. T. Millman, A. DeHaan, S. James, and K. D. Lissemore. 2010. Reduction in pain response by combined use of local lidocaine anesthesia and systemic ketoprofen in dairy calves dehorned by heat cauterization. Can. Vet. J. 51:283-288.

Espinoza, C., S. Lomax, and P. Windsor. 2013. The effect of a topical anesthetic on the sensitivity of calf dehorning wounds. J. Dairy Sci. 96:2894-2902. https://doi.org/10.3168/jds.2012-5954.

Faulkner, P. M., and D. M. Weary. 2000. Reducing pain after dehorning in dairy calves. J. Dairy Sci. 83:2037-2041. https://doi.org/10 .3168/jds.S0022-0302(00)75084-3.

Hannibal, K. E., and M. D. Bishop. 2014. Chronic stress, cortisol dysfunction, and pain: A psychoneuroendocrine rationale for stress management in pain rehabilitation. Phys. Ther. 94:1816-1825. https://doi.org/10.2522/ptj.20130597.

Heinrich, A., T. F. Duffield, K. D. Lissemore, and S. T. Millman. 2010. The effect of meloxicam on behavior and pain sensitivity of dairy calves following cautery dehorning with a local anesthetic. J. Dairy Sci. 93:2450-2457. https://doi.org/10.3168/jds.2009-2813.

Hempstead, M. N., J. R. Waas, M. Stewart, V. M. Cave, and M. A. Sutherland. 2018. Evaluation of alternatives to cautery disbudding of dairy goat kids using physiological measures of immediate and longer-term pain. J. Dairy Sci. 101:5374-5387.

Karlen, K. J., F. S. Baier, S. L. Odegard, R. M. Baumann, J. F. Coetzee, S. I. Kehoe, and K. D. Vogel. 2019. Efficacy of oral meloxicam as primary pain mitigation following caustic paste disbudding of three day old Holstein calves. Transl. Anim. Sci. https://doi .org/10.1093/tas/txz151.

Langbein, J., and M. L. Raasch. 2000. Investigations on the hiding behaviour of calves at pasture. Arch. Tierzucht 43:203-210.

Makimura, S., and N. Suzuki. 1982. Quantitative determination of bovine serum haptoglobin and its elevation in some inflammatory disease. Nihon Juigaku Zasshi 44:15-21. https://doi.org/10.1292/ jvms1939.44.15.

McGuirk, S. M. 2013. Calf Health Scorer. School of Veterinary Medicine, University of Wisconsin. Accessed December 11, 2019. https: //www.vetmed.wisc.edu/dms/fapm/apps/chs.htm.

McGuirk, S. M., and S. F. Peek. 2014. Timely diagnosis of dairy calf respiratory disease using a standardized scoring system. Anim. Health Res. Rev. 15:145-147. https://doi.org/10.1017/ S1466252314000267.

McMeekan, C. M., K. J. Stafford, D. J. Mellor, R. A. Bruce, R. N. Ward, and N. G. Gregory. 1998. Effects of regional analgesia and/ or a non-steroidal anti-inflammatory analesgic on the acute cortisol response to dehorning in calves. Res. Vet. Sci. 64:147-150. https://doi.org/10.1016/S0034-5288(98)90010-8.

Mellor, D. J., K. J. Stafford, S. E. Todd, T. E. Lowe, N. G. Gregory, R. A. Bruce, and R. N. Ward. 2002. A comparison of catecholamine and cortisol response of young lambs and calves to painful husbandry procedures. Aust. Vet. J. 80:228-233. https://doi.org/ 10.1111/j.1751-0813.2002.tb10820.x.

Milligan, B. N., T. F. Duffield, and K. D. Lissemore. 2004. The utility of ketoprofen for alleviating pain following dehorning in young dairy calves. Can. Vet. J. 45:140-143.

Mintline, E. M., M. Stewart, A. R. Rogers, N. R. Cox, G. A. Verkerk, J. M. Stookey, J. R. Webster, and C. B. Tucker. 2013. Play behaviour as an indicator of animal welfare: Disbudding in dairy calves. Appl. Anim. Sci. 144:22-30. https://doi.org/10.1016/j.applanim .2012.12.008.

Morisse, J. P., J. P. Cotte, and D. Huonnic. 1995. Effect of dehorning on behaviour and plasma cortisol responses in young calves. Appl. Anim. Behav. Sci. 43:239-247. https://doi.org/10.1016/0168 -1591(95)00569-E.

National Animal Health Monitoring System (NAHMS). 2014. Dairy 2014: Health and management practices on U.S. dairy operations. Accessed December 11, 2019. https://www.aphis.usda.gov/animal _health/nahms/dairy/downloads/dairy14/Dairy14_dr_PartIII .pdf.

National Farm Animal Care Council. 2009. Code of Practice for the Care and Handling of Dairy Cattle. Ottawa, Ontario, Canada.

O'Connor, A. 2010. Reporting guidelines for primary research: Saying what you did. Prev. Vet. Med. 97:144-149. https://doi.org/10 $.1016 / \mathrm{j}$.prevetmed.2010.09.010.

Petrie, N. J., D. J. Mellor, K. J. Stafford, R. A. Bruce, and R. N. Ward. 1996. Cortisol response of calves to two methods of disbudding used with or without local anesthetic. N. Z. Vet. J. 44:9-14 https://doi.org/10.1080/00480169.1996.35924.

Skinner, J. G., R. A. Brown, and L. Roberts. 1991. Bovine haptoglobin response in clinically defined field conditions. Vet. Rec. 128:147149. https://doi.org/10.1136/vr.128.7.147.

Stafford, K. J., and D. J. Mellor. 2005. Dehorning and disbudding distress and its alleviation in calves. Vet. J. 169:337-349. https:/ doi.org/10.1016/j.tvjl.2004.02.005. 
Stilwell, G., R. C. de Carvalho, M. S. Lima, and D. M. Broom . 2009. Effect of caustic paste disbudding, using local anesthesia with and without analgesia, on behavior and cortisol of calves. Appl. Anim. Sci. 116:35-44. https://doi.org/10.1016/j.applanim.2008.06.008.

Stilwell, G., M. S. Lima, and D. M. Broom. 2008. Comparing plasma cortisol and behaviour of calves dehorned with caustic paste after non-steroidal-anti-inflammatory analgesia. Livest. Sci. 119:63-69. https://doi.org/10.1016/j.livsci.2008.02.013.

Stilwell, G., M. S. Lima, R. C. Carvalho, and D. M. Broom. 2012. Effects of hot-iron disbudding, using regional anaesthesia with and without carprofen, on cortisol and behaviour of calves. Res. Vet. Sci. 92:338-341. https://doi.org/10.1016/j.rvsc.2011.02.005.

Stock, M. L., S. L. Baldridge, D. Griffin, and J. F. Coetzee. 2013. Bovine dehorning: Assessing pain and providing analgesic management. Vet. Clin. North Am. Food Anim. Pract. 29:103-133. https: //doi.org/10.1016/j.cvfa.2012.11.001.

Stock, M. L., L. A. Barth, N. K. Van Engen, S. T. Millman, R. Gehring, C. Wang, E. A. Voris, L. W. Wulf, L. Labeur, W. H. Hsu, and J. F. Coetzee. 2016. Impact of carprofen administration on stress and nociception responses of calves to cautery dehorning. J. Anim. Sci. 94:542-555. https://doi.org/10.2527/jas.2015-9510.

Stock, M. L., S. T. Millman, L. A. Barth, N. K. Van Engen, W. H. Hsu, C. Wang, R. Gehring, R. L. Parsons, and J. F. Coetzee. 2015. The effects of firocoxib on cautery disbudding pain and stress responses in preweaned dairy calves. J. Dairy Sci. 98:6058-6069. https://doi.org/10.3168/jds.2014-8877.

Sutherland, M. A., G. L. Lowe, F. J. Huddart, J. R. Waas, and M. Stewart. 2018. Measurement of dairy calf behavior prior to onset of clinical disease and in response to disbudding using automated calf feeders and accelerometers. J. Dairy Sci. 101:8208-8216. https: //doi.org/10.3168/jds.2017-14207.

Sutherland, M. A., D. J. Mellor, K. J. Stafford, N. G. Gregory, R. A. Bruce, and R. N. Ward. 2002. Cortisol responses to dehorn- ing of calves given a 5-h local anesthetic regimen plus phenylbutazone, ketoprofen, or adrenocorticotropic hormone prior to dehorning. Res. Vet. Sci. 73:115-123. https://doi.org/10.1016/S0034 $-5288(02) 00005-\mathrm{X}$.

Vickers, K. J., L. Niel, L. M. Kiehlbauch, and D. M. Weary. 2005. Calf response to caustic paste and hot-iron dehorning using sedation with and without local anesthetic. J. Dairy Sci. 88:1454-1459. https://doi.org/10.3168/jds.S0022-0302(05)72813-7.

Winder, C. B., C. A. Bauman, T. F. Duffield, H. W. Barkema, G. P. Keefe, J. Dubuc, F. Uehlinger, and D. F. Kelton. 2018. Canadian national dairy study: Heifer calf management. J. Dairy Sci. 101:10565-10579. https://doi.org/10.3168/jds.2018-14680.

Winder, C. B., S. J. LeBlanc, D. B. Haley, K. D. Lissemore, M. A Godkin, and T. F. Duffield. 2016. Practices for the disbudding and dehorning of dairy calves by veterinarians and dairy producers in Ontario, Canada. J. Dairy Sci. 99:10161-10173. https://doi.org/10 .3168/jds.2016-11270.

Winder, C. B., S. J. LeBlanc, D. B. Haley, K. D. Lissemore, M. A. Godkin, and T. F. Duffield. 2017. Clinical trial of local anesthetic protocols for acute pain associated with caustic paste disbudding in dairy calves. J. Dairy Sci. 100:6429-6411. https://doi.org/10 $.3168 /$ jds.2017-12724.

\section{ORCIDS}

Cassandra N. Reedman (ㄴ) https://orcid.org/0000-0003-3904-0993

Todd F. Duffield (ㅇ https://orcid.org/0000-0001-6035-4669

Trevor J. DeVries (ㄴ) https://orcid.org/0000-0001-9364-2456

Kerry D. Lissemore (ㄴ) https://orcid.org/0000-0003-2979-4708

Niel A. Karrow @ 1 https://orcid.org/0000-0003-2240-3022

Charlotte B. Winder ํ https://orcid.org/0000-0002-7314-3657 